Revue Française de Civilisation Britannique

\title{
Compte-rendu de Portraits of Violence : War and the Aesthetics of Disfigurement de Suzannah Biernoff
}

Sophie Sibson

\section{CpenEdition}

\section{Journals}

Édition électronique

URL : http://journals.openedition.org/rfcb/5093

DOI : $10.4000 /$ rfcb.5093

ISSN : 2429-4373

Éditeur

CRECIB - Centre de recherche et d'études en civilisation britannique

Référence électronique

Sophie Sibson, « Compte-rendu de Portraits of Violence: War and the Aesthetics of Disfigurement de Suzannah Biernoff », Revue Française de Civilisation Britannique [En ligne], XXIV-4 | 2019, mis en ligne le 23 novembre 2019, consulté le 27 novembre 2019. URL : http://journals.openedition.org/rfcb/5093 ; DOI : 10.4000/rfcb.5093

Ce document a été généré automatiquement le 27 novembre 2019.

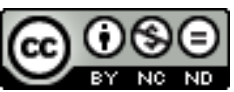

Revue française de civilisation britannique est mis à disposition selon les termes de la licence Creative Commons Attribution - Pas d'Utilisation Commerciale - Pas de Modification 4.0 International. 


\title{
Compte-rendu de Portraits of Violence : War and the Aesthetics of Disfigurement de Suzannah Biernoff
}

\author{
Sophie Sibson
}

\section{RÉFÉRENCE}

Biernoff, Suzannah. Portraits of Violence: War and the Aesthetics of Disfigurement, Ann

Arbor, Michigan : University of Michigan Press, 2017.

1 Suzannah Biernoff est actuellement maître de conférences en culture visuelle et contemporaine à Birkbeck, Université de Londres. Son domaine de recherche concerne l'histoire du corps et du visage, parallèlement aux cultures visuelles, à travers les périodes médiévales et modernes.

2 "Tout le monde voit quelque chose de différent"(p3). ${ }^{1}$ Voici ce qu'explore le livre de Suzannah Biernoff : Portraits of Violence: War and the Aesthetics of Disfigurement. Biernoff se concentre sur la culture visuelle et l'expérience des blessés de guerre.

3 L'auteur se penche sur la représentation des blessés de la face à travers une étude de la culture visuelle et non de l'art en tant que tel (p5). Biernoff inclut donc des objets visuels non artistiques (photographies médicales, coupures de journaux, moules de masques) mais les réactions de la population face aux blessés, défigurés, ou corps handicapés sont également étudiées (p5).

4 L'ouvrage se compose de cinq chapitres. Le premier est basé sur des photographies de soldats blessés de notre époque, afin d'examiner les conventions et les limites de portraits comme médiateurs des conséquences atroces de la guerre. Le deuxième chapitre analyse la stigmatisation du défigurement durant la Grande Guerre. À travers les chapitres III et IV, Biernoff analyse les photos et dessins de défigurés de la Première Guerre. Elle note ainsi le lien étroit entre illustrations médicales et portraits. Bien que ces œuvres ne fussent jamais conçues pour être exposées au public de l'époque, elles 
mettent en perspective le lien entre la science et l'art, la beauté et l'horreur. Le chapitre $\mathrm{V}$ est un retour au présent avec une étude sur la résurrection digitale d'un patient de Gillies ${ }^{2}$ dans le jeu vidéo Bioshock ${ }^{3}$. À travers ce chapitre Biernoff étudie la signification des représentations historiques dans l'art contemporain et digital.

Pour Biernoff, le défigurement devient un problème moral ou esthétique dans les yeux de l'autre. Afin de défendre ce point de vue, elle embarque le lecteur à travers différentes périodes allant de la Grande Guerre jusqu'à aujourd'hui, dans une exploration approfondie de photographies, de dessins et de peintures de soldats défigurés.

Dans notre société actuelle, la photographie est un des éléments clés dans la communication du conflit. En effet, à travers un portrait, nous pouvons mesurer la violence d'un conflit, en ressentir la souffrance (p37). Cependant est-ce que notre définition de la beauté s'en trouve modifiée ? À travers une étude de travaux d'artistes actuels tels que le photographe anglais Stuart Griffiths ${ }^{4}$ et la photographe Nina Berman, ${ }^{5}$ établie à New York, Suzannah Biernoff conclut que quand le visage d'une personne est altéré ou caché, quand l'image renvoyée par le visage est celle d'un traumatisme plus que celle d'une émotion, alors le portrait de cette personne perd de son identité aux yeux du public (p54).

7 Lorsque nous revenons à l'époque de la Grande Guerre, nous voyons que le défiguré est quasiment totalement absent de la sphère artistique visuelle en Grande-Bretagne (p56). Biernoff argumente que la raison de cela provient du fait que le soldat défiguré représentait un problème aux yeux du public car, contrairement aux amputés, le défiguré avait perdu toute humanité. L'auteur note alors l'importance de l'art dans le processus d'humanisation du défiguré, notamment à travers la création de masques. Cependant, il est difficile de connaître le réel impact de cet élan d'humanité sur les soldats eux-mêmes, ni même de savoir s'ils portaient effectivement leur masque ou encore s'ils furent réellement soulagés de leur apparence après quantité de chirurgies plastiques. Quant aux dessins biens connus produits par Henry Tonks ${ }^{6}$, Suzannah Biernoff souligne qu'ils furent créés dans un but médical et artistique. Par conséquent, il est extrêmement difficile de les interpréter clairement (p137). On peut dire que Biernoff a parfaitement mis en avant l'étroite relation entre l'histoire médicale et l'histoire de l'art. En effet, des dessins de Tonks émanent une certaine intimité mais aussi un recul médical qui rend le dessin dénué d'émotion?

8 Par ailleurs, Biernoff évoque un sujet qui, bien que déjà évoqué dans d'autres œuvres, mériterait une étude approfondie : la différence de perception du défiguré en France et en Angleterre. Pendant la Grande Guerre, environ $40 \%$ du contingent français furent blessés et entre 11 et $14 \%$ de ces derniers furent touchés au visage. Près de 15000 grands blessés de la face furent hospitalisés ${ }^{8}$. En France, des représentants des soldats défigurés furent présents lors de la signature du Traité de Versailles, ce qui, selon Biernoff, montre que les défigurés étaient gérés de manière collective (p113). 


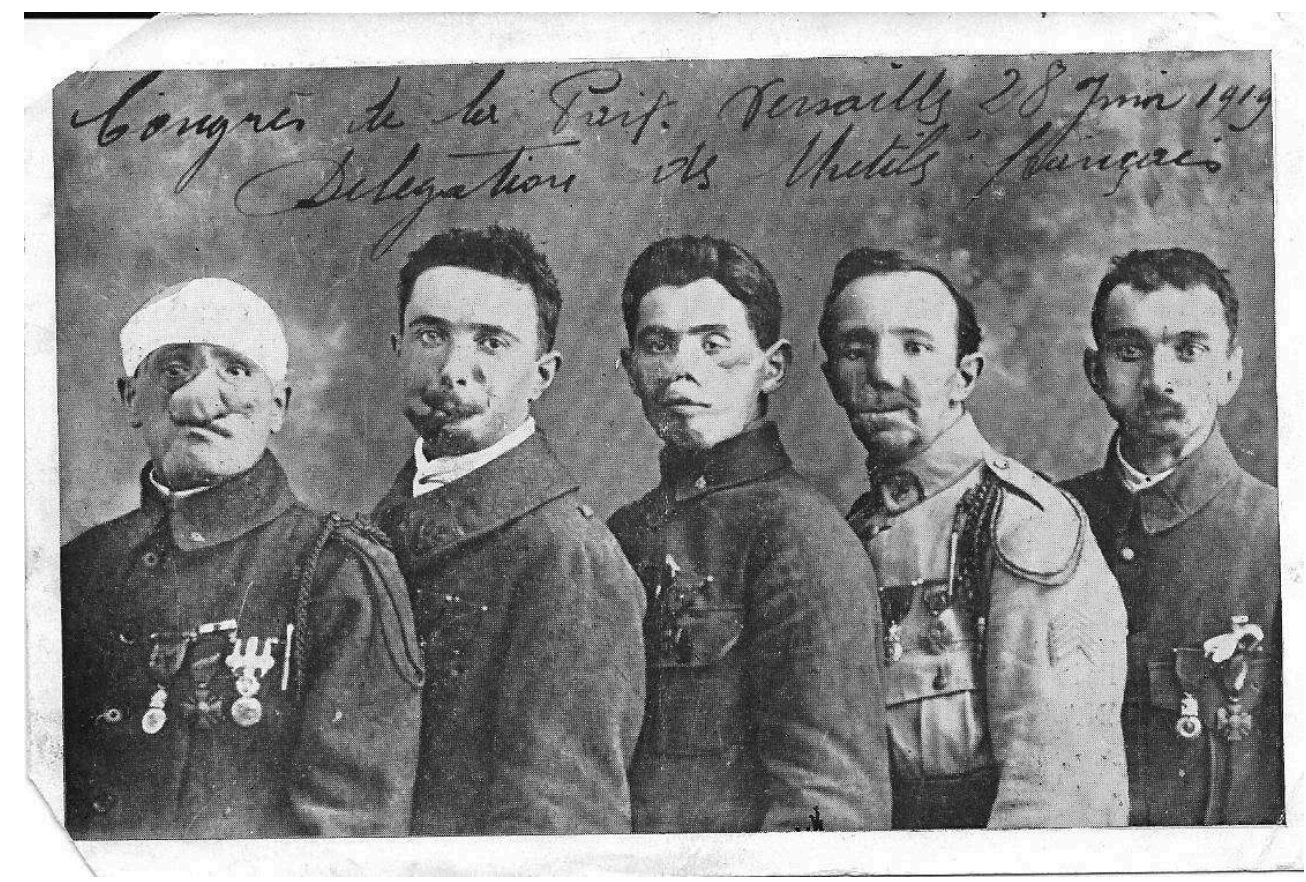

De gauche à droite : Eugène Hébert, Henri Agogué, Pierre Richard, Albert Jugon, André Cavalier. Les 5 " gueules cassées » présentes lors de la signature du Traité de Versailles.

Gelly, Georges. "Appel en faveur du foyer des Gueules cassées." (Paris : Société régionale d'imprimerie et de la publicité, 1926), p. 5.

Alors que nous pouvons considérer cette présence comme un acte positif, mettant à l'honneur ces hommes qui ont défendu leur pays, Andrew Bamji' adopte une vision plus négative. Il considère cela comme une exposition à l'intention des vaincus afin de leur montrer les conséquences de "leur guerre". Pour lui, si effectivement il n'y avait pas d'associations telles que les "Blessés de la Face" en Angleterre, cela venait du fait que les vétérans n'en ressentaient pas le besoin. Il affirme que le bon traitement des soldats défigurés à Sidcup, le suivi personnalisé des chirurgiens de chaque homme, les liens forts tissés entre les patients, les différents soins apportés aux soldats sur le site, sont tous des facteurs qui ont éliminé la nécessité de former une association pour venir en aide à ces hommes ${ }^{10}$. Même si fondamentalement les défigurés de guerre français comme anglais furent confrontés aux mêmes difficultés de réinsertion, force est de constater que ces derniers étaient beaucoup plus montrés dans l'espace public en France qu'en Grande-Bretagne.

10 Selon Biernoff, deux facteurs rentrent en jeu dans la perception horrifique de la défiguration par le peuple britannique. La première est que les images de sacrifice s'étaient toujours concentrées sur le visage comme symbole de pureté et de courage. Le deuxième facteur serait, selon Biernoff, la peur permanente de la syphilis qui était encore une malade incurable, associée à des lésions et difformités faciales. Une comparaison détaillée de la culture visuelle de ces deux pays face à ses défigurés pourrait être un élément clé dans la compréhension de l'attitude de chacun à la vue d'un visage défiguré.

11 Suzannah Biernoff n'insiste pas sur le sujet de la masculinité des années 1900, qui est dans un certain sens toujours présent dans notre société actuelle. Le lecteur aurait envie de lire davantage sur sa propre analyse de l'influence des idéaux masculins de l'époque sur la perception de la défiguration. Elle cite cependant à plusieurs reprises 
Dismembering The Male: Men's Bodies, Britain and the Great War ${ }^{11}$ de Joanna Bourke, mais note que les blessures de la face restent un sujet souvent mis à l'écart des discussions autour de la masculinité et des souffrances. Cette œuvre amène le lecteur à réfléchir à la définition de la beauté et à son impact sur autrui.

\section{BIBLIOGRAPHIE}

Bamji, Andrew. Faces From the Front. (Solihull : Helion and Company, 2017).

Biernoff, Suzannah. Portraits of Violence : War and the Aesthetics of Disfigurement. (Ann Arbor, Michigan : University of Michigan Press, 2017).

Bourke, Joanna. Dismembering the Male : Men's Bodies, Britain and the Great War. (Londres : Reaktion Books, 1996).

Messager, Danielle. Les "gueules cassées", pionniers sans visage de la médecine moderne. 26 octobre 2017. <https://www.franceinter.fr/societe/les-gueules-casses-ont-fait-avancer-la-medecine> [20 avril 2019]

Tonks, Henry. "The Tonks Pastels." Gillies archives.

<http://www.gilliesarchive.org.uk/Tonks\%20pastels/content.index.html> [13 août 2019]

\section{NOTES}

1. "Everyone sees something different."

2. Sir Harold Gillies (1882-1960) né en Nouvelle-Zélande mais ayant vécu à Londres est considéré comme le père fondateur de la chirurgie plastique moderne. Il exerça successivement à l'hôpital d'Aldershot puis au Queen Mary's Hospital de Sidcup qui ferma ses portes aux défigurés en 1925.

3. Jeu vidéo de tir à la première personne sorti en 2007.

4. Stuart Griffiths, né en 1972 à Manchester est un ancien soldat britannique maintenant photographe. Son premier livre The Myth of the Airborne Warrior fut publié en 2011 puis son deuxième Pigs Disco en 2013.

5. Nina Berman, née en 1960 à New York est une photographe documentaire américaine. Elle a publié trois livres Purple Hearts - Back from Iraq (2004), Homeland (2008) dont une autobiographie An Autobiography of Miss Wish (2017).

6. Henry Tonks (1862-1937) était un chirurgien britannique qui plus tard devint peintre et caricaturiste. Il est notamment connu pour ses dessins de soldats défigurés.

7. Tonks, Henry. "The Tonks Pastels." Gillies archives.

<http://www.gilliesarchive.org.uk/Tonks\%20pastels/content.index.html> [13 Août 2019]

8. Messager, Danielle. Les "gueules cassées", pionniers sans visage de la médecine moderne. 26 Octobre 2017. <https://www.franceinter.fr/societe/les-gueules-casses-ont-fait-avancer-la-medecine> [20 Avril 2019]

9. Andrew Bamji a travaillé en tant que médecin conseil en rhumatologie et réhabilitation à l'hôpital de Queen Mary à Sidcup de 1983 à 2011. Depuis 1989, Andrew Bamji s'intéresse aux dossiers médicaux des sections britanniques et néo-zélandaises qui travaillaient à Sidcup durant 
la Grande Guerre. Ces 2500 dossiers font partie des seules archives cliniques de ce conflit, elles incluent des notes chirurgicales, des diagrammes, des radios et des aquarelles.

10. Bamji, Andrew. Faces From the Front. (Solihull: Helion and Company , 2017), p. 114.

11. Bourke, Joanna. Dismembering the Male : Men's Bodies, Britain and the Great War. (Londres: Reaktion Books, 1996).

\section{AUTEURS}

SOPHIE SIBSON

Doctorante, travaillant sous la direction de John Mullen, Université de Rouen-Normandie 